\title{
Correspondence
}

\section{Vaccination and anesthesia: more questions than answers}

\author{
doi:10.1111/j.1460-9592.2007.02318.x
}

SIR-We found the survey by Short et al. in the May 2006 issue of Pediatric Anesthesia (1) very interesting and believe it opened some theoretical 'food for thought' about the perioperative behavior of the immunized child. However the authors conclusions and recommendations that elective surgery should be postponed for one week after killed vaccine and for three weeks after live-attenuated vaccine administration is a little too strong and the findings of their study cannot support such strong recommendations. The accompanying editorial (2) tried to address some of our concerns; however there is a need to expand on some of the controversies.

Vaccinations are among the most successful and costeffective public health tools. Their use has led to the global eradication of diseases such as small pox and near elimination of diseases like measles and polio in the Western hemisphere. The World Health Organization estimates that 2 million childhood deaths were prevented through vaccination in 2003 (3).

While the immunoparetic role of anesthesia and surgery are theoretical possibilities, there is incontrovertible evidence that immunization saves lives and childhood mortality in the prevaccination era and in countries with poorly organized immunization programs is appalling. To ask parents to postpone elective surgical procedures for a 3 week period after immunization may not be practical. One fundamental principle of active immunization is that continued exposure to the antigen is needed for adequate immune response hence the need for periodic booster doses (4). For example the 'triple vaccine' Diphtheria, Tetanus toxoid and acellular pertussis (DTP) and oral or inactivated polio vaccine are administered to children at 4weekly intervals between age $2-6 \mathrm{mo}$. The recommendation of Short et al. will be impractical in countries that predominantly use the oral (or live-attenuated) polio vaccine. The same arguments will apply to other liveattenuated vaccines such as measles, mumps, rubella (MMR) or Varicella.

Another important consideration in live-attenuated vaccine administration is the issue of household contacts. These can easily transmit the altered virus to contacts who can become actively immunized this way and may in fact develop vaccine-related illness (5). Should we then recommend that surgery be postponed in patients with positive contact history? Clearly this recommendation will escalate the number of unnecessary cancellations and more importantly increase the number of unimmunized children.

It is not clear from the literature whether the immunopertubation that results from anesthesia and surgery is a dose-effect relationship. Does long duration of surgery and anesthesia cause greater perturbation of the immune system or does short procedures like elective myringotomy, tonsillectomy or herniorrhaphy carry the same risk as say craniofacial reconstruction or open heart surgery? These are issues that need to be addressed before we can recommend postponing surgery in immunized children or postponing immunization in children with impending surgery.

Olubukola O Nafiu mD Frca
IAN Lewis mD FRCA
Division of Pediatric Anesthesiology,
Department of Anesthesiology,
University of Michigan Hospital 1500
East Medical Centre Drive,
Ann Arbor, MI, USA
(email: onafiu@med.umich.edu)

\section{References}

1 Short JA, Van der Walt JH, Zoanetti DC. Immunization and anesthesia - an International survey. Pediatr Anesth 2006; 16: 514-522.

2 Currie J. Vaccination: is it a real problem for anesthesia and surgery? Pediatr Anesth 2006; 16: 501-503.

3 WHO. 2004 Global Immunization Data.Available at http:// www.who.int/ immunization_monitoring/data/GlobalImmuni zationData.pdf.

4 King GE, Hadler SC. Simultaneous administration of childhood vaccines: an important public health policy that is safe and efficacious. Pediatr Infect Dis J 1994; 13: 394-407.

5 Fishman JA, Rubin RHInfection in organ-transplant recipients. N Engl J Med 1998; 338: 1741-1751.

\section{Author's reply}

doi:10.1111/j.1460-9592.2007.02321.x

We thank Drs Nafiu and Lewis for responding to our paper on immunization and anesthesia, as we believe this is an issue worthy of debate. They are concerned that the recommendations we made to postpone elective surgery for 1 week after inactive vaccines and 3 weeks after administration of live attenuated vaccines may not be practical and that the survey results we reported do not support them. 